\title{
The expression and role of serum response factor in papillary carcinoma of the thyroid
}

\author{
HEE JIN KIM ${ }^{1}$, KYUNG RYOUL KIM ${ }^{1,3,4}$, HO SUNG PARK ${ }^{1,2,4}$, KYU YUN JANG ${ }^{1,2}$, \\ MYOUNG JA CHUNG ${ }^{1,2}$, MINHO SHONG ${ }^{5}$ and WOO SUNG MOON ${ }^{1,2,3,4}$ \\ ${ }^{1}$ Department of Pathology, Chonbuk National University, Medical School, ${ }^{2}$ Institute for Medical Sciences, \\ ${ }^{3}$ Diabetes Research Center, ${ }^{4}$ The Center for Healthcare Technology Development, Jeonju, Chonbuk 561-756; \\ ${ }^{5}$ Department of Internal Medicine, Chungnam National University, Medical School, Dae Jeon, Chungnam 301-747, Korea
}

Received February 16, 2009; Accepted April 28, 2009

DOI: 10.3892/ijo_00000312

\begin{abstract}
Serum response factor (SRF) is a transcription factor of the MADS box family. SRF is involved in various cellular processes such as expression of immediate early and tissuespecific genes, cell proliferation, differentiation and apoptosis. The expression of SRF in papillary thyroid carcinoma (PTC) and its role have not been investigated, forming the basis for this study. Surgical specimens of 63 conventional PTCs along with 30 follicular adenoma, 30 adenomatous hyperplasia and 9 anaplastic carcinoma specimens were obtained from the surgical archives. The expression of SRF was determined by the use of immunohistochemical staining. We also investigated the expression level of SRF and an SRF target gene, $c$-fos in fresh PTC tissues and thyroid cancer cell lines (NPA, FRO and ARO) by Western blot analyses. In addition, we examined the role of SRF in PTC by overexpresion of SRF in the NPA cell line. SRF was mainly expressed in cancer cells, showing a strong nuclear and/or cytoplasmic staining in PTC. SRF was expressed in 50 of 63 cases of papillary carcinoma (79\%), 18 of 30 cases of follicular adenoma (60\%), 10 of 30 cases of nodular hyperplasia $(33 \%)$ and 6 of 9 cases of anaplastic carcinoma $(67 \%)$. The expression level of SRF was significantly up-regulated in PTC (combined staining score of 5.21 \pm 0.43$)$ and anaplastic carcinoma $(5.67 \pm 1.45)$ compared to that of follicular adenoma $(2.30 \pm 0.44)(\mathrm{P}<0.001)$, or adenomatous goiter $(1.13 \pm 0.28)(\mathrm{P}<0.001)$. Western blot analyses showed an increased expression of the spliced form of SRF protein and c-Fos protein in PTC as compared to non-tumor thyroid tissues. SRF expression correlated with the tumor size of the PTCs $(\mathrm{P}<0.05)$. Overexpression of SRF in papillary carcinoma cells enhanced cell motility and invasiveness. Our results indicate that the altered expression of
\end{abstract}

Correspondence to: Dr Woo Sung Moon, Department of Pathology, Chonbuk National University, Medical School, Jeonju, Chonbuk 561-756, Korea

E-mail: mws@chonbuk.ac.kr

Key words: carcinoma, metastasis, papillary, serum response factor, thyroid
SRF in papillary carcinoma cells may play an important role in PTC carcinogenesis and progression.

\section{Introduction}

Serum response factor (SRF) is a transcription factor of the MADS box family $(1,2)$. To date, DNA binding sites for SRFs (serum response elements-SREs) have been found in the promoters of $\sim 50$ different genes, including immediateearly genes such as c-fos and Egr-1 (3-5). Studies have also shown that SRF is involved in various cellular processes such as expression of immediate-early and tissue-specific genes, cell proliferation, differentiation and apoptosis (6-10). A previous study has demonstrated that SRF plays a role in tumor progression, specifically in the mesenchymal transition of epithelial tumor cells (11). We have recently reported that expression of SRF correlates with tumor cell dedifferentiation and enhances cell motility and invasiveness in hepatocellular carcinoma (HCC) cells (12).

Papillary thyroid carcinoma (PTC) is the most common thyroid cancer and the incidence of this disease has markedly increased in recent years. However, the expression of SRF in thyroid papillary carcinoma as well as its role has not been investigated. In the present study, we have investigated the expression and localization of SRF in papillary carcinoma, anaplastic carcinoma, adenomatous goiter and follicular adenoma specimens. We also examined the role of SRF in papillary carcinoma cells by overexpression of SRF.

\section{Materials and methods}

Patients and specimens. The Human Ethics Committee of Chonbuk National University Medical School approved this study. Paraffin-embedded thyroid specimens were obtained from the archival pathology data of the Department of Pathology at Chonbuk National University Hospital. A total of 132 selected specimens consisted of 63 conventional papillary carcinomas (PTCs), 30 follicular adenomas, 30 adenomatous goiters and 9 anaplastic carcinomas. All of the patients had undergone surgery and did not receive either chemotherapy or radiotherapy before surgical resection. Among the 63 PTCs studied, 41 were $>1 \mathrm{~cm}$ in diameter (mean size, $1.56 \mathrm{~cm}$ ) and 22 tumors were $<1 \mathrm{~cm}$ (mean size, $0.63 \mathrm{~cm}$ ). Eleven of 
63 patients were diagnosed with a lymph node metastasis Ten pairs of fresh PTC specimens and adjacent non-tumor thyroid tissues were obtained and were preserved in liquid nitrogen until use.

Thyroid cancer cell lines. The human thyroid cancer cell lines NPA (papillary carcinoma), FRO (follicular carcinoma) and ARO (anaplastic carcinoma) were purchased from the Korean Cell Line Bank (Seoul, Korea). These cell lines were cultured according to the recommendations of the cell bank. The cell lines were maintained in DMEM supplemented with $10 \%$ fetal bovine serum (FBS) without antibiotics. The cells were cultured in a $37^{\circ} \mathrm{C}$ incubator with an atmosphere of $5 \% \mathrm{CO}_{2}$.

Immunohistochemical analysis. For immunohistochemical staining of tissue samples, the Dako Envision system, that uses dextran polymers conjugated with horseradish peroxidase (Dako, Carpinteria, CA, USA) was performed, as described previously (12). The sections were incubated for $2 \mathrm{~h}$ at room temperature with anti-SRF (1:100 dilution; Santa Cruz Biotechnology, Santa Cruz, CA, USA). Peroxidase activity was detected with the enzyme substrate 3-amino-9-ethyl carbazole. For a negative control, the sections were treated in the same manner without the use of a primary antibody.

Interpretation of SRF immunoreactivity. Two independent pathologists who had no knowledge of the histopathological data scored the expression of SRF and a high level of concordance $(95 \%)$ was achieved. The samples that were subjected to immunostaining were rated according to a score calculated by multiplying the intensity of the stain to the area of the stain. The intensity of cell staining was graded according to the following scale: 0 , no staining; $1+$, mild staining; $2+$, moderate staining; $3+$ strong staining. The area of staining was evaluated using the following scale: $0,0-19 \%$ of the cells stained positive; $1+, 20-39 \%$ of the cells stained positive; $2+, 40-69 \%$ of the cells stained positive; $3+,>70 \%$ of the cells stained positive. The maximum combined score was 9 and the minimum score was zero.

Transfection of SRF complementary DNA. Human SRF complementary DNA (cDNA) (accession number: J03161) was cloned into pcDNA3 (Invitrogen, Carlsbad, CA, USA). The same plasmid without the SRF DNA insert was used as a control vector. All plasmids were amplified in DH5 $\alpha$ Escherichia coli competent cells (Invitrogen) and were purified using an endo-free plasmid mega-prep kit (Qiagen, Valencia, CA, USA). Transient transfection of NPA, FRO and ARO cells with human SRF or vector cDNA was performed using Lipofectamin (Gibco BRL, Gaithersburg, MD, USA).

Protein extraction and determination of the SRF and c-Fos protein levels by Western blotting. Thyroid papillary carcinoma tissues were homogenized using an Ultra-Turrax homogenizer (Ika, Staufen, Germany) in ice-cold lysis buffer (50 mM Tris, pH 7.5, $150 \mathrm{mM} \mathrm{NaCl}, 0.5 \%$ Nonidet P-40) containing $1 \mathrm{mM}$ phenylmethylsulfonyl fluoride, $2 \mu \mathrm{g} / \mathrm{ml}$ leupeptin, $2 \mu \mathrm{g} / \mathrm{ml}$ aprotinin, $5 \mathrm{mM}$ sodium fluoride and $1 \mathrm{mM}$ sodium orthovanadate. The lysates were subjected to Western blotting, as described previously (12). Since SRF is known to regulate expression of genes such as $c-F o s$, the expression levels of c-Fos and SRF protein were evaluated in an SRFtransfected cell line and thyroid papillary carcinoma tissues. Bound antibody signals were visualized by the use of a chemiluminescence system (Amersham Life Sciences, Arlington Heights, IL, USA). The membranes were stripped and were reprobed with a monoclonal anti- $\beta$-actin antibody (Sigma, St. Louis, MO, USA) to normalize protein loading. Quantification of the data was performed using ImageQuant software (Molecular Dynamics, Piscataway, NJ, USA).

In vitro assays of cell migration and invasion. A cell migration assay was performed using Transwell chambers, as described previously (12). The cells transfected with either the SRF expression plasmid or the control vector plasmid were serum starved for $48 \mathrm{~h}$. Transwell chambers equipped with $8 \mu \mathrm{m}$ Matrigel-coated filters (24-well format) (Becton-Dickinson, Franklin Lakes, NJ, USA) were rehydrated and $4 \times 10^{4}$ cells in $400 \mu 1$ of serum-free DMEM were seeded in the upper chamber. DMEM containing 2 or $5 \%$ fetal bovine serum (FBS) was used in the lower chamber. After incubation for 12-22 $\mathrm{h}$ at $37^{\circ} \mathrm{C}$, cells on the upper surface of the filter were mechanically removed with a cotton swab. The filters were fixed and were stained using a Diff-Quick staining kit (Dade Behring, Newark, DE, USA). The cells on the lower surface of filters were counted under a light microscope at X100 magnification in five randomly selected fields per well. The migration assay was also performed with Transwell chambers where the filters were not coated with Matrigel using the same procedure. The experiment was repeated independently three times.

Statistical analysis. Data are expressed as means \pm SE. The differences in the numerical data between groups were evaluated by use of the Mann-Whitney $U$ test. Associations between the expression of SRF and clinicopathological factors were tested by use of Chi-squared test. The following clinicopathological factors were correlated with SRF expression: age and gender, lymph node metastasis and size of tumor $(<1 \mathrm{~cm}$ vs. $\geq 1 \mathrm{~cm})$. Comparisons between groups were performed using the Student's t-test for the migration and invasion assays. A P-value $<0.05$ was considered as statistically significant.

\section{Results}

Overexpression of SRF in thyroid carcinoma specimens. The results of immunohistochemical analysis are summarized in Table I. When a uniform cut-off of $20 \%$ was chosen, SRF was overexpressed in 50 of 63 papillary carcinomas (79\%), 6 of 9 anaplastic carcinomas $(67 \%), 18$ of 30 follicular adenomas $(60 \%)$ and 10 of 30 adenomatous hyperplasia (33\%), respectively. The mean scores for immunohistochemical staining of SRF from papillary carcinoma or anaplastic carcinoma samples were significantly higher than the mean scores for follicular adenoma or adenomatous hyperplasia samples (Table II). When a uniform cut-off of $10 \%$ was chosen, we also obtained a significant difference in the expression of SRF between the malignant and benign lesions (data not shown). Representative immunostaining of tissue samples is shown in Fig. 1. SRF was mainly expressed 

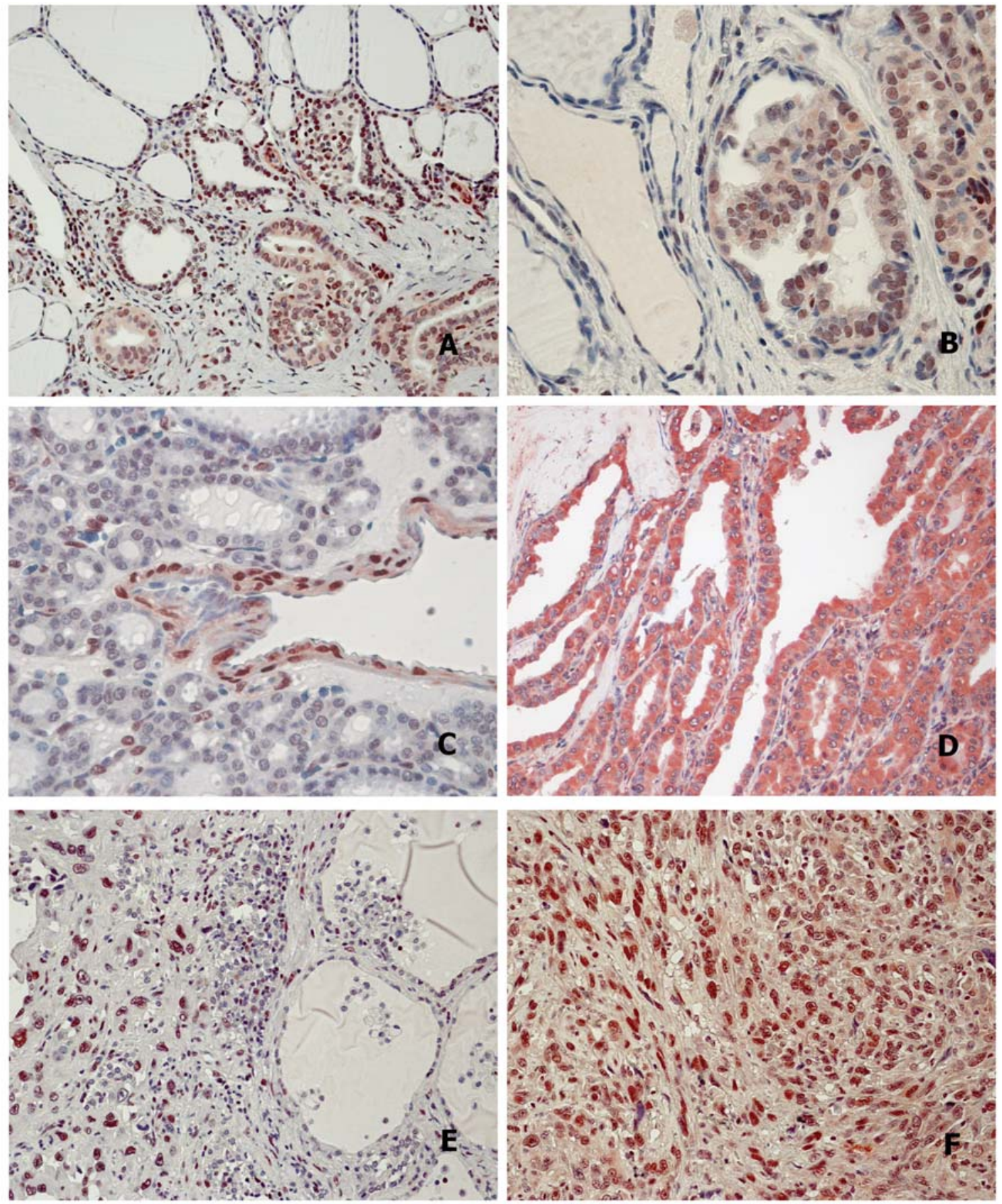

Figure 1. Immunohistochemical expression of SRF. (A and B) Papillary carcinoma cells showed strong positivity for SRF in the nuclei of tumor cells, while non-malignant follicular cells were negative for SRF expression. (C) Follicular adenoma cells showed minimal expression of SRF in the nuclei of tumor cells. Note the strong nuclear expression of SRF in the smooth muscle cells of blood vessel (as internal positive control). (D) Predominant cytoplasmic expression of SRF is seen in papillary carcinoma cells. (E and F) Strong nuclear expression of SRF is seen in anaplastic carcinoma cells.

Table I. Summary of the results of immunohistochemical staining for SRF in various thyroid lesions.

\begin{tabular}{|c|c|c|c|c|c|c|c|c|}
\hline \multirow[b]{2}{*}{ Pathology } & \multirow[b]{2}{*}{ Positive cases $(\%)$} & \multicolumn{4}{|c|}{ SRF-positive cells (\%) } & \multicolumn{3}{|c|}{ Intensity } \\
\hline & & $<20 \%$ & $20-39 \%$ & $40-69 \%$ & $>70 \%$ & $1+$ & $2+$ & $3+$ \\
\hline Papillary carcinoma $(\mathrm{n}=63)$ & $50(79.3)$ & 13 & 4 & 7 & 39 & 8 & 19 & 23 \\
\hline Anaplastic carcinoma $(n=9)$ & $6(66.6)$ & 3 & 0 & 0 & 6 & 0 & 1 & 5 \\
\hline Follicular adenoma $(\mathrm{n}=30)$ & $18(60)$ & 12 & 7 & 6 & 5 & 9 & 8 & 1 \\
\hline Adenomatous hyperplasia $(n=30)$ & $10(33.3)$ & 20 & 8 & 2 & 0 & 0 & 10 & 0 \\
\hline
\end{tabular}


Table II. Comparison of the expression level of SRF in various thyroid lesions.

Immunohistochemical

SRF scoring (mean $\pm \mathrm{SE})$

\begin{tabular}{ll}
\hline Papillary carcinoma $(\mathrm{n}=63)$ & $5.21 \pm 0.43$ \\
Anaplastic carcinoma $(\mathrm{n}=9)$ & $5.67 \pm 1.45$ \\
Follicular adenoma $(\mathrm{n}=30)$ & $2.30 \pm 0.44^{\mathrm{a}}$ \\
Adenomatous goiter $(\mathrm{n}=30)$ & $1.13 \pm 0.28^{\mathrm{a}}$ \\
\hline
\end{tabular}

Statistical analysis performed by use of the Mann-Whitney U test. ${ }^{\text {a }}>0.001$ compared to score of papillary carcinoma.

Table III. Relationship between SRF expression in PTC and tumor size or the presence of a lymph node metastasis.

A, SRF expression

\begin{tabular}{lcrc}
\hline & \multicolumn{3}{c}{ Tumor size } \\
\cline { 2 - 4 } & $>1 \mathrm{~cm}$ & $<1 \mathrm{~cm}$ & Total \\
\hline SRF-positive & $38(60 \%)$ & $16(25 \%)$ & $54(86 \%)$ \\
SRF-negative & $3(5 \%)$ & $6(10 \%)$ & $9(14 \%)$ \\
Total & $41(65 \%)$ & $22(35 \%)$ & $63(100 \%)$
\end{tabular}

Statistical analysis performed by use of the Chi-squared test. $\mathrm{P}=0.031$.

B, Lymph node metastasis

\begin{tabular}{lcrc}
\hline & $\begin{array}{c}\text { Positive } \\
\text { lymph node }\end{array}$ & $\begin{array}{c}\text { Negative } \\
\text { lymph node }\end{array}$ & Total \\
\hline SRF-positive & $10(16 \%)$ & $44(70 \%)$ & $54(86 \%)$ \\
SRF-negative & $1(2 \%)$ & $8(12 \%)$ & $9(14 \%)$ \\
Total & $11(17 \%)$ & $52(83 \%)$ & $63(100 \%)$ \\
\hline
\end{tabular}

Statistical analysis performed by use of the Chi-squared test. $\mathrm{P}=0.587$.

in cancer cells with strong nuclear and/or cytoplasmic staining in PTC samples (Fig. 1A). No or minimal staining was observed in normal thyroid follicular cells (Fig. 1B). In addition to the strong staining observed in carcinoma cells, smooth muscle cells of large vessels (as an internal positive control) and occasional stromal cells and endothelial cells showed positive nuclear staining for SRF (Fig. 1C). Among 50 positive cases of PTCs, 22 cases showed nuclear and cytoplasmic staining, 15 showed predominantly nuclear staining and the remaining 13 samples showed cytoplasmic staining only (Fig. 1D). All of the positive cases of the anaplastic carcinomas demonstrated nuclear staining only (Fig. 1E and F). None of the non-cancerous thyroid tissue samples showed cytoplasmic staining. The SRF expressing cells in papillary carcinoma and anaplastic carcinoma were usually $>70 \%$ of the total tumor cells and showed strong intensity.

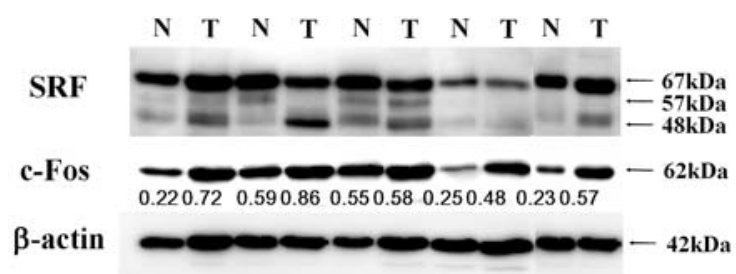

Figure 2. Western blot analysis of SRF and c-Fos in papillary thyroid carcinoma (PTC) tissues. The use of Western blotting demonstrated increased expression of spliced variants of SRF in PTC tissues or a different ratio of full-length SRF protein to spliced variants of SRF as compared to the corresponding non-tumor thyroid tissues.

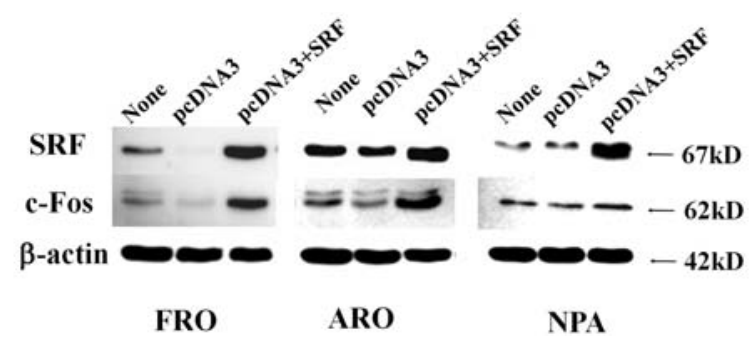

Figure 3. Enhanced expression of SRF and c-Fos proteins in thyroid carcinoma cells transfected with the SRF gene. Western blot analysis showed a marked increase in the protein level of c-Fos in the SRF-transfected thyroid carcinoma cells.

Expression of SRF in follicular adenoma or adenomatous hyperplasia was usually $<40 \%$ of total tumor cells and showed a low expression level. Large papillary carcinomas $(\geq 1 \mathrm{~cm})$ had significantly higher SRF expression than small-sized $(<1 \mathrm{~cm})$ tumors (Table III). There was no close correlation between the level of SRF expression and gender or age. Although there was no statistically significance between SRF expression in papillary carcinomas and the presence of a lymph node metastasis, 10 of 11 papillary carcinoma (91\%) specimens with a lymph node metastasis displayed strong SRF expression, indicating an important role of SRF in the formation of lymph node metastases.

Expression of SRF and c-Fos in thyroid papillary carcinoma tissues. We evaluated the expression of SRF and c-Fos in 10 pairs of PTC tissues and corresponding non-tumor thyroid tissue. Representative SRF and c-Fos expression is shown in Fig. 2. Western blot analyses showed increased expression of spliced variants of SRF in PTC tissues or a different ratio of full-length SRF protein to spliced variants of SRF as compared to the corresponding non-tumorous thyroid tissues. SRF $\Delta 45$ was the predominant isoform expressed in PTC tissue. The density for c-Fos against $\beta$-actin showed a significant increase of c-Fos in tumor samples as compared to non-tumor tissues (Fig. 2).

Overexpression of SRF increases expression of c-Fos. To verify the above observations that SRF is overexpressed in thyroid cancer tissues, we examined SRF expression levels in the NPA (papillary carcinoma), FRO (follicular carcinoma) and ARO (anaplastic carcinoma) cell lines. Western blot analyses revealed a different level of SRF expression in each cell line (Fig. 3). As compared to the SRF expression level of 
A

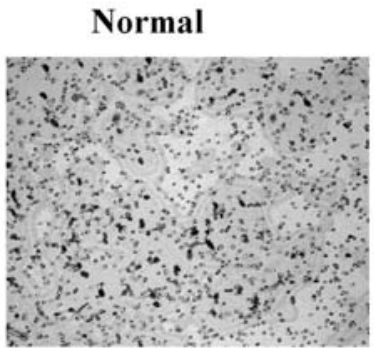

Mock

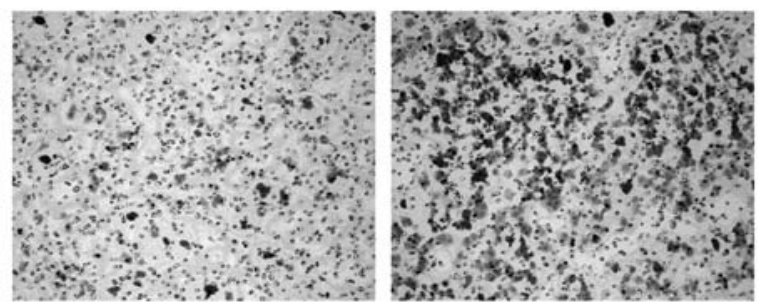

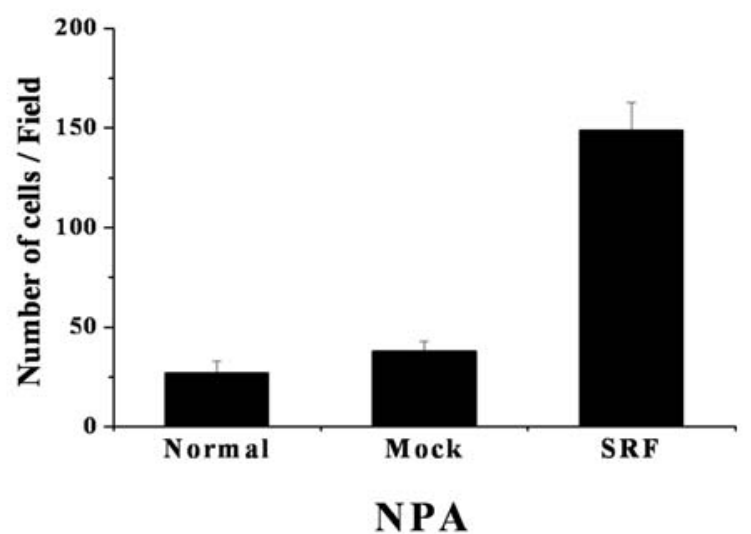

B
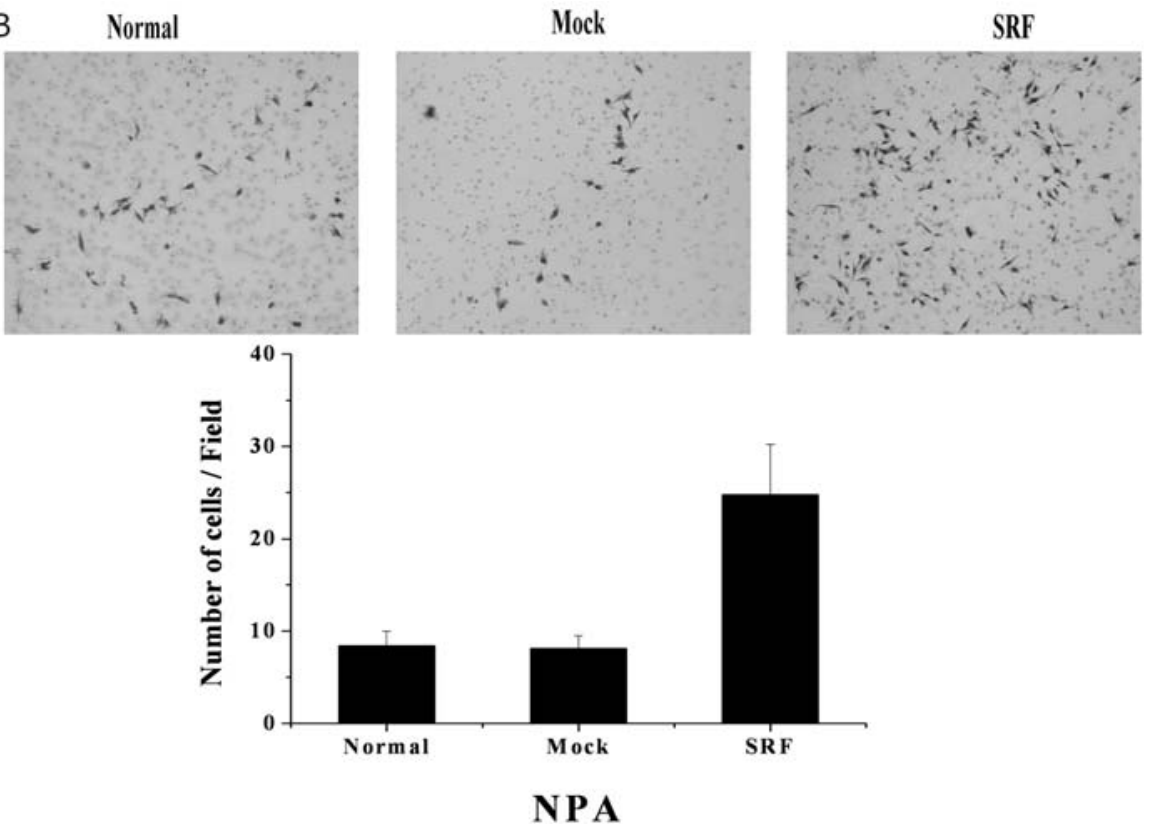

Figure 4. In vitro cell migration and invasion assay. (A) SRF-transfected NPA (papillary carcinoma) cells showed 3.3-fold increased migration with the use of the Boyden chamber assay. (B) In SRF-transfected NPA cells, cell invasion was increased by 2.1-fold when compared with the use of non-transfected control cells.

NPA and FRO cells, the SRF expression level was higher in ARO cells. We also analyzed the expression levels of SRF and an SRF target gene, $c$-fos, in SRF-transfected NPA, FRO and ARO cells. The use of Western blotting confirmed upregulation of SRF production in cells transiently transfected with SRF cDNA. In SRF-transfected NPA, FRO and ARO cells, expression levels of SRF were increased by 2.7-, 7.3- and 2.1-fold, respectively. Expression levels of c-Fos were also increased by 1.5-, 3.3- and 3.8-fold, respectively (Fig. 3).

SRF increases thyroid cancer cell migration and invasion. To determine the role of SRF in thyroid cancer cell migration and invasion, we performed a cell migration and invasion assay. Overexpression of SRF in NPA cells increased cell migration by 3.3-fold as compared to cell migration of control cells (Fig. 4A). Overexpression of SRF also increased cell invasion by 2.1 -fold as compared to cell invasion of control cells (Fig. 4B) $(\mathrm{P}<0.05)$.

\section{Discussion}

This study demonstrated for the first time the following. a) SRF is significantly up-regulated in PTC and anaplastic carcinoma as compared to SRF expression in follicular 
adenoma or adenomatous goiter; b) Western blot analyses show a different expression level of the spliced form of SRF protein and increased expression of c-Fos protein in PTC as compared to expression in non-tumor thyroid tissues; c) SRF expression correlates with tumor size of PTC; d) overexpression of SRF in the thyroid cancer cells enhances the expression of c-Fos; e) 10 of 11 papillary carcinoma (91\%) specimens with a lymph node metastasis display strong SRF expression. Furthermore, overexpression of SRF in papillary carcinoma cells enhanced cell motility and invasiveness. These findings clearly indicate the important role of SRF in PTC development and progression.

SRF plays a key role in many cellular processes, including cell cycle regulation, apoptosis, cell growth and differentiation and cell-specific gene regulation (3-10). Accumulating evidence has suggested that SRF plays multiple roles in carcinogenesis and cancer metastasis. The SRF DNA binding activity correlates with increased expression of SRF and squamous epithelial tumor progression, specifically at the transition to an invasive metastatic stage of carcinogenesis (11). We have also shown that expression of SRF correlates with tumor cell dedifferentiation and that overexpression of SRF enhances cell motility and invasiveness of HCC cells (12). In the present study, we found that expression of SRF as well as c-Fos protein is increased in PTC as compared to non-tumor thyroid tissues. Moreover, overexpression of SRF in thyroid cancer cells enhanced the expression level of c-Fos protein, cell migration and invasiveness. In accord with our findings, a previous study has demonstrated that c-Fos expression is significantly up-regulated in thyroid cancer patients (13). c-Fos, an immediate early gene, is one of the SRF targets and is activated by a variety of growth factors and mitogens through several different signaling pathways (14). Indeed, c-Fos has been implicated in the most fundamental processes occurring in mammalian cells such as cell cycle control, (15) cell differentiation, (16) oncogenic transformation and tumor progression $(17,18)$. Together, these findings indicate that up-regulation of SRF and c-Fos expression promotes tumor progression and metastasis of PTC.

Little is known about the expression or role of alternatively spliced isoforms of SRF during cancer development and progression. Previously, Patten et al have demonstrated that the SRF $\Delta 5$ isoform is overexpressed in a human colon cancer cell line derived from a poorly differentiated tumor (19). These investigators have also suggested that SRF $\Delta 5$ contributes to epithelial tumorigenesis through the inhibition of apoptosis. Expression of SRF 45 is enhanced in human hypoplastic lungs whereas the spliced form is suppressed during normal bronchial myogenesis (20). In addition, Yang et al have shown that in bleomycin-exposed mouse lungs, the level of SRF $\Delta 5$ is increased mainly due to de novo synthesis (21). They have also observed translocation of SRF/SRF $\Delta 5$ into the nuclei from the cytoplasm during the development of myofibroblasts in bleomycin-induced lung injury. Although the antibody used in this study does not distinguish between SRF and its isoforms, our results have demonstrated the presence of nuclear and/or cytoplasmic localization of SRF in PTC cells. We also found increased expression of spliced variants of SRF, predominantly the SRF $\triangle 45$ form, in PTC tissue as compared to the corresponding non-tumor thyroid tissue. These findings suggest that a change in expression of the alternatively spliced isoforms of SRF is closely related to tumorigenesis of PTC. Elucidation of how the alternatively spliced isoforms of SRF regulates cellular function will be valuable in the understanding of how SRF contributes to the tumorigenesis of PTC.

A number of different substances have been evaluated as potential markers in the differential diagnosis of thyroid neoplastic lesions. In this study, we showed significant differences in the expression of SRF among normal thyroid tissue, adenomatous goiter, follicular adenoma and papillary carcinoma and up-regulation for the lymph node metastases of papillary carcinoma. However, there is considerable overlap in SRF immunoreactivity between those with benign lesions and PTC. Although SRF is of potential use in the differential diagnosis of PTC and benign nodular lesions, a combination of other immunohistochemical markers with a careful morphological assessment is recommended.

In conclusion, our results indicate that SRF is significantly up-regulated in PTC and anaplastic carcinoma. Moreover, overexpression of SRF in thyroid cancer cells enhances the expression level of the SRF target protein, c-Fos and is associated with tumor progression and metastasis. In addition, we have found a different expression level of the spliced form of SRF in PTC tissues. These findings indicate that altered expression of SRF may have an important role in tumorigenesis and progression of PTC.

\section{Acknowledgements}

We thank Professor Mie-Jae Im for her discussion and editorial suggestions. This study was supported by a grant of the Korean Ministry of Education, Science and Technology (The Regional Core Research Program/The Center for Healthcare Technology Development) and by the Ministry of Science and Technology $(\mathrm{MoST}) /$ Korea Science and Engineering Foundation (KOSEF) through the Diabetes Research Center at Chonbuk National University (R13-2008-005-0000-0).

\section{References}

1. Treisman R: Identification of a protein-binding site that mediates transcriptional response of the c-fos gene to serum factors. Cell 46: 567-574, 1986.

2. Shore $P$ and Sharrocks AD: The MADS-box family of transcription factors. Eur J Biochem 229: 1-13, 1995.

3. Norman C, Runswick M, Pollock R and Treisman R: Isolation and properties of cDNA clones encoding SRF, a transcription factor that binds to the c-fos serum response element. Cell 55: 989-1003, 1998.

4. Sobue K, Hayashi K and Nishida W: Expressional regulation of smooth muscle cell-specific genes in association with phenotypic modulation. Mol Cell Biochem 190: 105-118, 1999.

5. Chai J and Tarnawski AS: Serum response factor: discovery, biochemistry, biological roles and implications for tissue injury healing. J Physiol Pharmacol 53: 147-157, 2002.

6. Schratt G, Weinhold B, Lundberg AS, et al: Serum response factor is required for immediate-early gene activation yet is dispensable for proliferation of embryonic stem cells. Mol Cell Biol 21: 2933-2943, 2001.

7. Camoretti-Mercado B, Liu HW, Halayko AJ, et al: Physiological control of smooth muscle-specific gene expression through regulated nuclear translocation of serum response factor. $\mathrm{J}$ Biol Chem 275: 30387-30393, 2000.

8. Ding W, Gao S and Scott RE: Senescence represses the nuclear localization of the serum response factor and differentiation regulates its nuclear localization with lineage specificity. J Cell Sci 114: 1011-1018, 2001 
9. Bertolotto C, Ricci JE, Luciano F, et al: Cleavage of the serum response factor during death receptor-induced apoptosis results in an inhibition of the c-FOS promoter transcriptional activity. J Biol Chem 275: 12941-12947, 2000.

10. Zhang X, Chai J, Azhar G, et al: Early postnatal cardiac changes and premature death in transgenic mice overexpressing a mutant form of serum response factor. J Biol Chem 276: 40033-40040, 2001.

11. Psichari E, Balmain A, Plows D, Zoumpourlis V and Pintzas A: High activity of serum response factor in the mesenchymal transition of epithelial tumor cells is regulated by RhoA signaling. J Biol Chem 277: 29490-29495, 2002.

12. Park MY, Kim KR, Park HS, et al: Expression of the serum response factor in hepatocellular carcinoma: Implications for epithelial-mesenchymal transition. Int J Oncol 31: 1309-1315, 2007.

13. Kataki A, Sotirianakos S, Memos N, et al: P53 and C-FOS overexpression in patients with thyroid cancer: an immunohistochemical study. Neoplasma 50: 26-30, 2003.

14. Muller R, Bravo R, Burckhardt J and Curran T: Induction of c-fos gene and protein by growth factors precedes activation of c-myc. Nature 312: 716-720, 1985.

15. Kovary K and Bravo R: The jun and fos protein families are both required for cell cycle progression in fibroblasts. Mol Cell Biol 11: 4466-4472, 1991.
16. Lord KA, Abdollahi A, Hoffman-Liebermann B and Liebermann DA: Proto-oncogenes of the fos/jun family of transcription factors are positive regulators of myeloid differentiation. Mol Cell Biol 13: 841-851, 1993.

17. Mejlvang J, Kriajevska M, Berditchevski F, et al: Characterization of E-cadherin-dependent and -independent events in a new model of c-Fos-mediated epithelial-mesenchymal transition. Exp Cell Res 313: 380-393, 2007

18. Saez E, Rutberg SE, Mueller E, et al: c-fos is required for malignant progression of skin tumors. Cell 82: 721-732, 1995.

19. Patten LC, Belaguli NS, Baek MJ, et al: Serum response factor is alternatively spliced in human colon cancer. J Surg Res 121: 92-100, 2004

20. Yang Y, Beqaj S, Kemp P, Ariel I and Schuger L: Stretch-induced alternative splicing of serum response factor promotes bronchial myogenesis and is defective in lung hypoplasia. J Clin Invest 106: 1321-1330, 2000

21. Yang Y, Zhe X, Phan SH, Ullenbruch $M$ and Schuger L: Involvement of serum response factor isoforms in myofibroblast differentiation during bleomycin-induced lung injury. Am J Respir Cell Mol Biol 29: 583-590, 2003. 